\title{
Eventos clínicos adversos en el ámbito hospitalario: caracterización desde el punto de vista del profesional sanitario
}

\author{
Adverse clinical events in the hospital: characterization from the point of view \\ of the health worker
}

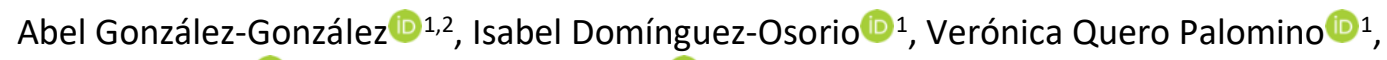
Magdalena León Velasco ${ }^{1}$, Filomena Polo Montes ${ }^{\circledR 1}$, Ximena Tatiana Lasso Ruales ${ }^{3}$, Lidia Martín Alonso ${ }^{3}$.

${ }^{1}$ Hospital General Universitario de Ciudad Real, España

Universidad de Castilla-La Mancha, España

${ }^{3}$ Hospital Universitario de Guadalajara, España

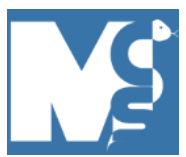

Recibido: $29 / 11 / 2021$

Revisado: 30/12/2021

Aceptado: 02/01/2022

\section{Autor correspondiente}

Abel González-González Hospital General Universitario de Ciudad Real, España

abelg@sescam.jccm.es

\section{Conflictos de interés}

Los autores declaran no poseer conflictos de interés.

\section{Fuente de financiación}

Los autores no recibieron apoyo financiero del Gobierno Regional de Tumbes para realizar esta investigación.

Este artículo es publicado bajo una licencia de Creative Commons Reconocimiento 4.0 Internacional.

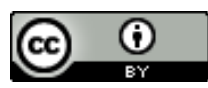

\section{RESUMEN}

Introducción: un evento adverso es un incidente que ha producido daño en un paciente y que puede o no estar asociado a un error clínico. Un porcentaje elevado de profesionales de la salud se encuentran implicados en este tipo de eventos anualmente. Gran parte de los profesionales desconocen la existencia de los sistemas implantados en sus centros de trabajo para una comunicación correcta de los eventos adversos. Objetivos: identificar las características de los profesionales hospitalarios afectados por algún evento adverso y evaluar su conocimiento de los sistemas de notificación. Metodología: estudio observacional descriptivo transversal. Se evaluaron variables demográficas, laborales y relacionadas con los eventos adversos y los sistemas de notificación. Resultados: participaron $207,45,4 \%$ enfermeros y $54,6 \%$ médicos, de $39,24 \pm 10,07$ años y con una experiencia de $14,43 \pm 9,9$ años. El $71,8 \%$ resultaron afectados por algún evento adverso. El primer evento adverso ocurrió a los 6,45 $\pm 6,18$ años. El 42,1 \% conocía algún sistema de notificación; pero sólo el $24,61 \%$ de éstos, afectados por un evento adverso, lo notificaron. Los médicos fueron más afectados por un evento adverso, además, más precozmente $(p<0,001)$; y también conocían los sistemas de notificación $(p=0,001)$. Conclusión: muchos sanitarios, con escasa experiencia, fueron afectados por eventos adversos. Pocos conocían los sistemas de notificación y muchos menos los utilizaban. Los médicos resultaron más afectados, y más precozmente, por los eventos adversos y conocían mejor los sistemas de notificación.

Palabras clave: Daño al paciente; eventos adversos; seguridad del paciente; personal sanitario.

\section{ABSTRACT}

Introduction: an adverse event is an incident that has caused harm to a patient and that may or may not be associated with a clinical error. A high percentage of health professionals are involved in this type of event annually. A large part of the professionals are unaware of the existence of the systems implemented in their work centers for a correct communication of adverse events. Objective: to identify the characteristics of hospital professionals affected by an adverse event and to evaluate their knowledge of the notification systems. Methods: cross-sectional descriptive observation-al study. Demographic, labor and related variables with adverse events and notification systems were evaluated. Results: $207,45.4 \%$ nurses and $54.6 \%$ doctors, participated, $39.24 \pm 10.07$ years and with an experience of $14.43 \pm 9.9$ years. $71.8 \%$ were affected by some adverse event. The first adverse event occurred at $6.45 \pm 6.18$ years. $42.1 \%$ knew of some notification system; but only $24.61 \%$ of these, affected by an adverse event, report-ed it. Physicians were more affected by an adverse event, moreover earlier $(p<0.001)$ and they were also more aware of notification systems $(p=0.001)$. Conclusions: many health workers, with little experience, were affected by adverse events. Few knew notification systems and far fewer used them. Doctors were more affected, and earlier, by adverse events and were better acquainted with notification systems. 


\section{INTRODUCCIÓN}

La Organización Mundial de la Salud define un incidente relacionado con la seguridad del paciente como "cualquier evento o circunstancia que ha ocasionado un daño innecesario a un paciente o podría haberlo hecho"; definición similar, prácticamente, a la que propone para este concepto, a nivel nacional, la norma UNE (Una Norma Española): “acontecimiento o situación imprevista o inesperada que puede producir, o no, daño al paciente" $(1,2)$. Cuando ante un incidente se ha producido daño; que puede ser daño temporal, permanente o muerte y que puede o no estar asociado a un error clínico, se habla de Evento Adverso (EA) (3). Datos recientes de España estiman que el $15 \%$ de los profesionales de la salud se encuentran implicados en este tipo de eventos anualmente y el $90 \%$ de estos mismos profesionales consideran que en su centro de trabajo hay una insuficiente ayuda y apoyo a los profesionales tras un EA $(4,5)$.

Existe una obligación legal y ética de informar de forma honesta a los pacientes ante un efecto adverso, pero muchos profesionales temen que hacerlo desemboque en una demanda por malpraxis (6). Gran parte de los profesionales desconocen la existencia de los sistemas implantados en sus centros de trabajo para una comunicación correcta de los EA. Explorar el ambiente laboral más habitual en el que ocurren estos EA; así como el grado de conocimiento que poseen los profesionales sanitarios de los Sistemas de Notificación (SN) de éstos puede tener una crucial importancia en la mejora del sistema sanitario. Además, es muy importante la implantación de programas de intervención para apoyar y formar a los trabajadores sanitarios tras un EA, porque los recursos humanos son el patrimonio más importante de las infraestructuras sanitarias $(7,8)$.

Con estos antecedentes, los objetivos de este trabajo de investigación fueron: investigar las circunstancias laborales de un grupo de trabajadores sanitarios implicados en un EA sufrido por un paciente, para intentar identificar posibles factores precipitantes prevenibles y evaluar el grado de conocimiento y utilización de los sistemas de notificación de los EA por parte de estos profesionales.

\section{METOdOLOGÍA}

Estudio observacional descriptivo transversal basado en las respuestas a una encuesta anónima, diseñada a propósito, entre los profesionales sanitarios (médicos y enfermeros) del Hospital General Universitario de Ciudad Real (España). El periodo de recogida de los datos fue de 7 meses: entre el 1 de abril y el 30 de octubre de 2020.
La muestra la constitutyen todos los profesionales de enfermería y medicina, entre 22 y 65 años, que trabajaban en las Unidades médicas y quirúrgicas del hospital durante el periodo de recogida de datos (muestreo consecutivo). Criterios de inclusión: Todos los médicos y enfermeros que trabajaban en las Unidades médicas y quirúrgicas del hospital que aceptaron participar en este estudio; sin ningún motivo de exclusión, salvo la no aceptación a hacerlo.

Las variables evaluadas fueron recopiladas en unos Cuadernos de Recogida de datos (CRD) diseñados específicamente para este estudio. Las variables utilizadas en este trabajo fueron agrupadas en 2 bloques: bloque I, datos demográficos y laborales; a saber: edad, sexo, tiempo de ejercicio profesional, unidad de hospitalización en la que se trabajaba (en el caso de enfermería) o especialidad (en el caso de medicina) y modalidad laboral (turnicidad, para enfermería; realización de guardias, para medicina) y bloque 2, datos relacionados con el evento adverso (EA), que incluían 6 preguntas: si había conocido algún EA que le hubiera afectado directamente; si era así, en qué tipo de jornada ocurrió (en el caso de enfermería, en qué turno de trabajo y en el de medicina si fue en jornada normal o durante una guardia); qué consecuencias tuvo el EA para el paciente (daño temporal, permanente o muerte); cuánto tiempo llevaba trabajando cuando ocurrió el EA (en caso de existir más de uno se refería sólo al primero) y finalmente; 2 preguntas relativas a los sistemas de notificación de los EA im-plantados en el hospital (SiNASP, Sistema de Notificación y Aprendizaje para la Seguridad del Paciente y SENSAR, Sistema Español de Notificación y Seguridad en Aneste-sia y Reanimación): Si conocían la existencia de estos sistemas y, en caso afirmativo, si los habían utilizado ante un EA.

Las variables se resumieron mediante estadísticos descriptivos adecuados a la natura-leza de cada variable: medidas de tendencia central (media) y de dispersión (desviación estándar) para las variables cuantitativas y frecuencias absolutas y relativas (porcentajes) para las cualitativas. La comparación entre las variables se realizó con la prueba Chi cuadrado cuando ambas eran cualitativas y con la prueba $t$ de Student cuando una de ellas era cuantitativa. El nivel de error alfa o significación estadística escogido fue inferior al $5 \%$.

\section{RESULTADOS}

Durante el año 2020, periodo de recogida de los datos de este trabajo, la plantilla de trabajadores activos en el hospital en el que se llevó a cabo fue de 500 médicos y de 1006 enfermeros. Cumplimentaron el CRD 207 
trabajadores sanitarios, 94 (el 45,4 \%) graduados en enfermería y 113 (el 54,6\%) graduados en medicina. Su media de edad fue de $39,24 \pm 10,07$ años y su distribución por sexos fue: 136 mujeres (el 65,7\%) y 71 hombres (el $34,3 \%$ ). El tiempo de ejercicio profesional en el momento de la recogida de datos fue de 14,43 \pm 9,9 años. Trabajaban por turnos o realizaban guardias médicas 181 sanitarios (el 90,95\% de los 199 que respondieron a esta cuestión). Desarrollaban su actividad profesional en unidades médicas 176 (el 85,8 $\%$ ) y 29 (el 14,1 \%) en qui-rúrgicas ( 2 trabajadores no contestaron). En la Tabla 1 se detallan todos los datos so-ciodemográficos y laborales de los participantes distribuidos por profesión.

TABLA 1: DISTRIBUCIÓN, POR PROFESIÓN, DE LOS DATOS SOCIODEMOGRÁFICOS Y LABORALES DE LA MUESTRA.

\begin{tabular}{lccc}
\hline & Enfermería & Medicina & $p$ \\
\hline Edad (años). Media \pm DE & $40 \pm 11,2$ & $38,61 \pm 9,04$ & 0,029 \\
Ejercicio profesional (años). Media \pm DE & $16,53 \pm 10,8$ & $12,85 \pm 8,86$ & 0,012 \\
Mujer. N (\%). & $76(80,85 \%)$ & $60(53,09 \%)$ & $<0,001$ \\
Modalidad laboral ${ }^{1}$. N (\%) & $78(87,6 \%)^{2}$ & $103(93,6 \%)^{3}$ & 0,143 \\
Unidad médica/quirúrgica ${ }^{4} . N(\%)$ & $80(87 \%) / 12(13 \%)^{5} 96(85 \%) / 17(15 \%)$ & 0,683
\end{tabular}

DE: Desviación estándar. N: frecuencia absoluta. ${ }^{1}$ Modalidad laboral: turnicidad, en el caso de personal de enfermería y realización de guardias, en el de personal médico. ${ }^{2}$ No contestaron 5 personas. El valor porcentual se refiere únicamente al total de los profesionales que han contestado (89). ${ }^{3}$ No contestaron 3 personas. El valor porcentual se refiere únicamente al total de los/as profesionales que han contestado (110). ${ }^{4}$ Unidad en la que trabajan en el caso de personal de enfermería y especialidad, en el caso de personal médico. ${ }^{5} \mathrm{No}$ contestaron 2 personas. El valor porcentual se refiere únicamente al total de los profesionales que han contestado (92).

A la pregunta de si habían conocido algún EA que les hubiera afectado directamente respondieron 206 profesionales; sólo un enfermero no contestó. Lo hicieron afirmativamente 148 (el 71,8\%): 94 mujeres (el $69,62 \%$ de ellas) frente a 54 hombres (el $76,5 \%$ de ellos). Sobre el tipo de jornada que estaban realizando en el momento en el que ocurrió el EA contestaron 142 (el $95,94 \%$ de los afectados por un EA): 88 de ellos (el
$61,97 \%)$ estaban en turnos de tarde o noche, en el caso de enfermería, o realizan-do una guardia, en el de medicina. Sobre las consecuencias del EA informaron 141 , el $95,27 \%$ de los afectados por uno de ellos. En la Figura 1 se presenta gráficamente la distribución porcentual de las consecuencias de los EA según el grado de gravedad.

\section{FIGURA 1: DISTRIBUCIÓN PORCENTUAL DE LAS CONSECUENCIAS PARA EL PACIENTE DEL EVENTO ADVERSO.}

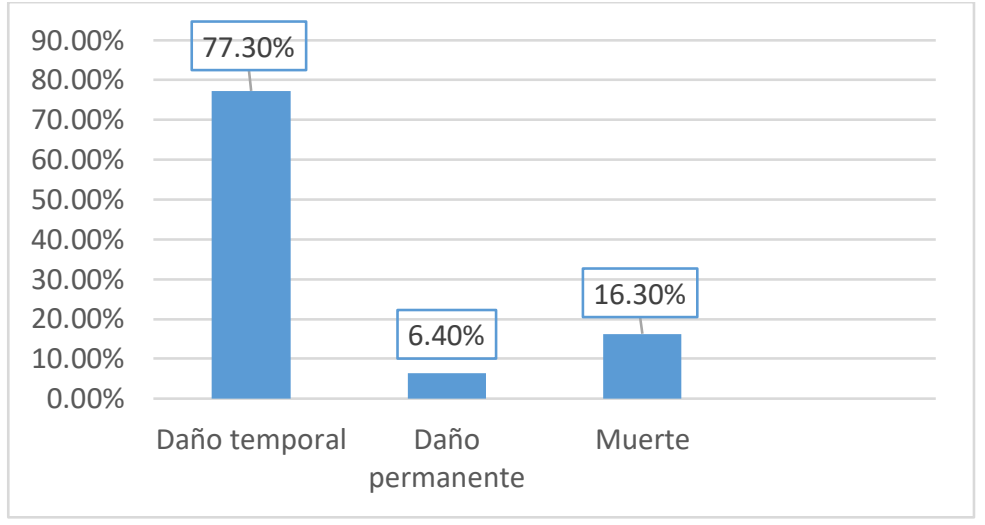

La media de tiempo de ejercicio profesional hasta que ocurrió el primer EA fue de 6,45 $\pm 6,18$ años. En 73 trabajadores (el $49,32 \%$ de los que fueron afectados) el primer EA ocurrió antes de los 5 años de ejercicio profesional. A la pregunta sobre el conocimiento de los sistemas de notificación de EA existentes en el hospital (SiNASP y/o SENSAR) contestaron 190 (el 91,8 \% de la muestra total). Reconocieron conocer alguno de ellos 80 profesionales, el 42,1\%: 51 mujeres (el $41,46 \%$ de las mujeres que contestaron a la pregunta) y 29 hombres (el 43,28\%). De los 80, 65 habían sido afectados por un EA; aunque sólo 16 de ellos (el 24,61 $\%)$ notificaron el EA a través de alguno de estos sistemas. Un mayor porcentaje de médicos reconocieron haber sido afectados por un EA y serlo de forma más precoz (en cuanto al tiempo de ejercicio profesional hasta el EA) que los enfermeros, a un nivel estadísticamente significativo $(p<0,001)$; también los médicos demostraron un mayor conocimiento de los sistemas de notificación de EA $(p=0,001)$ (Tabla 2). 
TABLA 2: DATOS RELACIONADOS CON LOS EVENTOS ADVERSOS Y CON EL CONOCIMIENTO DE LOS SISTEMAS DE NOTIFICACIÓN DE ÉSTOS, DISTRIBUIDOS POR PROFESIÓN.

\begin{tabular}{|c|c|c|c|}
\hline & Enfermería & Medicina & $p$ \\
\hline Afectado por algún EA. N (\%) & $55(59,13 \%)$ & $93(82,3 \%)$ & $<0,001$ \\
\hline Tipo de jornada: TT+TN (Enf). Guardia (Med). N (\%) & $31(59,61 \%)^{1}$ & $57(63,3 \%)^{2}$ & 0,660 \\
\hline Consecuencias del EA (DT/DP/M). N (\%) & $\begin{array}{c}40(75,47 \%) \\
5(9,43 \%) \\
8(15,9 \%)^{3}\end{array}$ & $\begin{array}{c}69(78,4 \%) \\
4(5,54 \%) \\
15(17,04 \%)^{4}\end{array}$ & $\begin{array}{l}0,509 \\
4\end{array}$ \\
\hline
\end{tabular}

Tiempo de ejercicio profesional hasta el primer EA (años).Media $\pm D E \quad 7,91 \pm 7,54 \quad 5,59 \pm 5,09<0,001$

Conocimiento de los sistemas de Notificación de los EA. N (\%) $\quad 24(28,91 \%)^{5} 56(52,33 \%)^{6} 0,001$

\begin{abstract}
EA: evento adverso. N: frecuencia absoluta. TT: turno de tarde. TN: turno de noche. DT: daño temporal. DP: daño permanente. M: muerte. DE: desviación estándar.13 enfermeros no explicitaron cuando ocurrió el EA. El valor porcentual se refiere únicamente al total de los profesionales que han contestado (52). 23 médicos no explicitaron cuando ocurrió el EA. El valor porcentual se refiere únicamente al total de los profesionales que han contestado (90).32 enfermeros no respondieron a esta pregunta. El valor porcentual se refiere únicamente al total de los profesionales que han contestado (53). 45 médicos no respondieron a esta pregunta. El valor porcentual se refiere únicamente al total de los profesionales que han contestado (88). 511 enfermeros no contestaron a esta pregunta. El valor porcentual se refiere únicamente al total de los profesionales que han contestado (83).66 médicos no respondieron a esta pregunta. El valor porcentual se refiere únicamente al total de los profesionales que han contestado (107).
\end{abstract}

\section{DISCUSIÓN}

La calidad asistencial va indisolublemente unida a la seguridad del paciente, por lo que reducir al mínimo posible los EA debe ser un objetivo prioritario de cualquier sistema de salud que busque la excelencia. En este sentido, parece imprescindible identificar las circunstancias laborales de los profesionales que rodean a los EA de forma más frecuente y promover el conocimiento y utilización de los SN de EA en los centros sanitarios (9).

Casi tres cuartas partes de los profesionales que respondieron a las preguntas del CRD (el 71,8 \%) reconocieron haber sido afectado por algún EA a lo largo de su vida profe-sional. Aunque este estudio no fue diseñado para evaluar la prevalencia de este hecho, y por lo tanto la muestra no puede ser objetivamente considerada como representati-va por el método de elección de ésta, el hecho de que un alto porcentaje de trabajado-res de la plantilla hospitalaria participase (el $22,6 \%$ de los médicos -113 de $500-$, sobre todo, pero también el 9,34 \% -94 de 1006- de enfermeros) aporta consistencia al dato de "posible prevalencia"; que se encontraría dentro del intervalo notificado en estudios recientes similares a éste $(10,11)$, así como en otros llevados a cabo con grandes muestras poblacionales (12); y que son superiores a los datos de prevalencia recogidos en una revisión sistemática publicada en 2013 (13). En el hospital en el que se realizó este trabajo, el porcentaje de médicos afectados por, al menos, un EA, por encima del $80 \%$, fue significativamente mayor que el de enfermeros.

Las características demográficas de las muestras poblacionales estudiadas en otros trabajos son muy variables; sin embargo, en algunos de ellos los rangos de edad $y$, sobre todo, de tiempo de ejercicio profesional son similares a los de los participantes de este trabajo $(10,14)$; lo que puede aportar más solidez a los resultados de las posibles comparaciones que puedan establecerse con algunos de los resultados encontrados por otros autores. Frente al personal médico, el de enfermería era, mayoritariamente mujer, de mayor edad y llevaba más tiempo trabajando, lo que probablemente es un reflejo de la estructura demográfica de ambas profesiones en el hospital.

Todo el personal sanitario es susceptible de verse afectado por un EA $(15,16,17)$. Sin embargo, gran parte de las investigaciones se han centrado en torno a las experiencias personales directas de EA en la figura del médico (18). En la literatura revisada se aprecia una gran variabilidad de resultados entre las distintas especialidades médicas $(19,20)$. En el presente estudio se buscó la participación de médicos y enfermeros de diferentes especialidades y unidades asistenciales.

La participación de los trabajadores de las unidades médicas del hospital, médicos y enfermeros, fue claramente mayor que la de las quirúrgicas, un dato que se interpreta como el reflejo del mayor peso, en cuanto a volumen de personal, de las primeras, porque los CRD fueron distribuidos por todas las unidades sin excepción; aunque no se evaluó el porcentaje de respuesta en cada unidad participante respecto a los CRD distribuidos.

Entre los resultados de este estudio sobresale el de que el $62 \%$ de los profesionales encuestados notificaron que el EA tuvo lugar en periodos temporales en los que el número de profesionales trabajando es menor, que 
son en los turnos de tarde-noche en el caso de enfermería y en el turno de guardia en el de medicina. Múltiples factores pueden contribuir a este mayor riesgo de los incidentes en la seguridad del paciente en estos periodos tiempo: la falta de personal, sin duda es una de ellas, pero también el exceso de trabajo, el estrés y la fatiga, probablemente derivados del primero. Pero, también, la explicación puede estar, simplemente, en que el periodo de tiempo considerado es más amplio que el correspondiente al turno de mañana de los días laborables, que es el único en que la actividad hospitalaria es plena, y por lo tanto la posibilidad de que el EA ocurra es mayor. Hay que tener en cuenta que además suelen atribuirse errores a la complejidad del trabajo, a la falta de cooperación entre equipos, a la mala continuidad de la atención, a fallas latentes de los sistemas, a insuficiencia de conocimiento o habilidad y a una supervisión inadecuada, aspectos, la mayoría, no evaluados en este trabajo $(21,22)$.

Respecto a las consecuencias de los EA, que habitualmente se gradúan por la grave-dad que tienen estos en el paciente, fueron más frecuentes los de carácter leve o temporal, coincidiendo con lo hallado por otros autores $(4,5)$; en este caso sin diferen-cias entre el personal médico y el de enfermería. En lo que difieren los datos publicados por distintos grupos es respecto a la dependencia entre la severidad del EA y el impacto psicológico en los profesionales de la salud $(23,24)$.

El espacio temporal hasta el suceso del primer EA se sitúa, en muchos casos, en los primeros 5 años de profesión: casi el $50 \%$ de los participantes en este estudio lo situa-ron antes de este límite (25). Estos datos apoyarían la posible relación de una menor experiencia por parte del personal sanitario con la probabilidad de ocurrencia de un EA. Entre el personal médico que participó en esta investigación el primer EA tuvo lugar antes que entre el personal de enfermería.

Finalmente, fue muy llamativo el escaso conocimiento entre los profesionales sanitarios de los SN de EA (menos del $50 \%$ ), aunque con una clara diferencia a favor de los médicos que casi duplicaron en este aspecto a los enfermeros. Pero es, si cabe, más destacable el paupérrimo uso que se hizo de estos SN, aun conociéndolos (menos del $25 \%$ ).

Estos datos podrían ser explicados por una ausencia de factores organizativos eficientes en las estructuras sanitarias. En los últimos años se ha realizado un esfuerzo informativo en el hospital en el que se llevó a cabo el estudio desde distintas estructuras (Dirección Gerencia, Comisión Central de Garantía de la Calidad y
Comité SiNAPS) que no parece haber dado el resultado esperado. En muchos de los ámbitos laborales descritos en la bibliografía no existía un sistema de información bien definido (8), y en aquellos en los que estaba presente, como podría ser el caso de este estudio, la cultura de temor al litigio y las dudas respecto a un posible apoyo satisfactorio se convierten en importantes factores limitantes del proceso de notificación (10).

El hecho de que la elección de la muestra poblacional no fuera realizada aleatoriamente es, sin duda, la mayor debilidad de este trabajo; pero como se ha explicado en el segundo párrafo de esta discusión, el alto porcentaje de participación mitiga en parte esta debilidad. La existencia de escasos trabajos de las mismas características que éste y el hecho de que los CRD fueron entregados individualmente a cada participante por parte de los autores del trabajo, permitiendo "in situ" la aclaración de las dudas sobre su cumplimentación y, por tanto, contribuyendo a aportar solidez a los resultados, son dos de las fortalezas de este estudio.

En conclusión; un porcentaje elevado de profesionales sanitarios habían conocido algún EA, la gran mayoría durante el primer decenio de actividad laboral. Pocos trabajadores conocían los sistemas de notificación de EA y muchos menos hacían uso de ellos. Lo médicos resultaron más afectados por un EA, además de más precozmente y demostraron un mayor conocimiento de los SN de éstos que los enfermeros. Son necesarios futuros estudios basados en la elección de muestras poblacionales aleatorizadas, con un tamaño suficiente para permitir obtener resultados que discriminen entre las distintas unidades y en la inclusión de profesionales de atención primaria que permitan confirmar y ampliar resultados como los aquí presentados.

\section{CONTRIBUCIÓN DE LOS AUTORES}

AGG ha participado en la concepción, diseño del trabajo, obtención de resultados, análisis de interpretación de los datos, redacción del manuscrito, revisión crítica del manuscrito y aprobación de su versión final; IDO y VQO han participado en la obtención de resultados, análisis de interpretación de los datos, redacción del manuscrito, revisión crítica del manuscrito y aprobación de su versión final; MLV y FPM han participado en el diseño del trabajo, en la obtención de resultados, en la revisión crítica del manuscrito y en la aprobación de su versión final y XTLR y LMA han participado en el diseño del trabajo, el análisis de interpretación de los datos, la revisión crítica del manuscrito y la aprobación de su versión final. 


\section{REFERENCIAS}

1. Rocco C, Garrido A. Seguridad del paciente y cultura de seguridad. Rev. Med. Clin. Condes. 2017; 28(5) 785-795. https://doi.org/10.1016/j.rmclc.2017.08.006

2. Cuadros K, Grillo K, Toffoletto MC, Henriquez-Roldán C, Canales MA. Ocurrencia de Incidentes de Seguridad del Paciente y Carga de Trabajo de Enfermería. Rev. Latino-Am. Enfermagem 2017;25:e2841. https://doi.org/10.1590/1518-8345.1280.2841

3. Rodziewicz TL, Houseman B, Hipskind JE. Medical Error Reduction and Preven-tion. 2021 Aug 6. In: StatPearls [Internet]. Treasure Island (FL): StatPearls Publish-ing; 2021. URL.

4. Mira JJ, Lorenzo S, Carrillo I, et al. Interventions in health organisations to reduce the impact of adverse events in second and third victims. BMC Health Serv Res. 2015; 341. https://doi.org/10.1186/s12913-015-0994-x

5. Aranaz JM, Mira JJ, Guilabert M, Herrero JF, Vitaller J, et al. Repercusión de los eventos adversos en los profesionales sanitarios. Estudio sobre las segundas víctimas. Trauma Fund MAPFRE 2013; 24 (1): 54-60. URL.

6. Burlison JD, Scott SD, Browne EK, Thompson SG, Hoffman $J M$. The second victim experience and support tool (SVEST): Validation of an organizational resource for assessing second victim effects and the quality of support resources. J Patient Saf. 2017; 13(2): 93-102. https://doi.org/10.1097/PTS.0000000000000129

7. Van Gerven E, Bruyneel L, Panella M, Euwema $M$, Sermeus W, Vanhaecht K. Psychological impact and recovery after involvement in a patient safety incident: a repeated measures analysis. BMJ Open 2016;6: e011403. https://doi.org/10.1136/bmjopen-2016-011403

8. Carrillo I, Ferrús L, Silvestre C, et al. Propuestas para el estudio del fenómeno de las segundas víctimas en España en atención primaria y hospitales. Rev Calid Asist. 2016; 31(S2):3-10. https://doi.org/10.1016/i.cali.2016.04.008

9. Wu AW. Medical error: the second victim. The doctor who makes the mistake needs help too. BMJ 2000; 320:726-7. https://doi.org/10.1136/bmj.320.7237.726

10. Harrison R, Lawton R, Stewart K. Doctors' experiences of adverse events in secondary care: the professional and personal impact. Clin Med 2014; 14: 585-90. https://doi.org/10.7861/clinmedicine.14-6-585

11. Edrees H, Connors C, Paine L, Norvell M, Taylor H, Wu AW. Implementing the RISE second victim support programme at the Johns Hopkins Hospital: a case study. BMJ Open 2016; 6(9): e011708. https://doi.org/10.1136/bmjopen2016-011708

12. Waterman AD, Garbutt J, Hazel E et al. The emotional impact of medical errors on practicing physicians in the United States and Canada. Jt Comm J Qual Patient Saf. 2007; 33:467-76. https://doi.org/10.1016/s15537250(07)33050-x

13. Seys D, Wu AW, Gerven EV, et al. Health care professionals as second victims after adverse events: a systematic review. Eval Health ProF. 2013; 36:135-62. https://doi.org/10.1177/016327871245891

14. Scott SD, Hirschinger LE, Cox KR, McCoig M, Brandt J, Hall LW. The natural history of recovery for the healthcare provider "second victim" after adverse patient events. Qual Saf Health Care 2009; 18:325-33. https://doi.org/10.1136/qshc.2009.032870

15. Saura J, Martínez A, Leal M, Gómez J. Percepción de los residentes de medicina familiar sobre sus errores clínicos tras dos años de formación. SEMERGEN. 2011; 37(6): 280286 https://doi.org/10.1016/j.semerg.2010.12.013

16. Treiber LA, Jones JH. Making an infusion error: the second victims of infusion therapy-related medication errors. J Infus Nurs. 2018;41 (3):156-163. https://doi.org/10.1097/NAN.0000000000000273

17. Cabilan CJ, Kynoch K. Experiences of and support for nurses as second victims of adverse nursing errors: a qualitative systematic review. JBI Database System Rev Implement Rep. 2017;15(9):2333-2364. https://doi.org/10.11124/JBISRIR-2016-003254

18. O'Connor E, Coates HM, Yardley IE, Wu AW. Disclosure of patient safety incidents: a comprehensive review. Int J Qual Health Care 2010; 22(5): 371-379. https://doi.org/10.1093/intqhc/mzq042

19. Han K, Bohnen JD, Peponis T et al. The Surgeon as the second victim? Results of the boston intraoperative adverse events surgeons' attitude (BISA) study. J Am Coll Surg. 2017; 224(6):1048-1056. https://doi.org/10.1016/j.jamcollsurg.2016.12.039

20. Leape LL, Brennan TA, N Laird et al. The nature of adverse events in hospitalized patients. Results of the Harvard Medical Practice Study II. N Engl J Med . 1991; 324(6):37784. https://doi.org/10.1056/NEJM199102073240605

21. Shor S, Tal O, Maymon R. The second victim: treating the health care providers. Harefuah. 2017;156(1):38-40. URL.

22. Makary MA, Daniel M. Medical error-the third leading cause of death in the US. BMJ. 2016;353: i2139. https://doi.org/10.1136/bmj.i2139

23. Vanhaecht K, Seys D, Schouten L, et al. Duration of second victim symptoms in the aftermath of a patient safety incident and association with the level of patient harm: a cross-sectional study in the Netherlands. BMJ open 2019; 9(7): e029923. https://doi.org/10.1136/bmjopen-2019$\underline{029923}$

24. Pyo J, Choi EY, Lee W, et al. Physicians' Difficulties Due to Patient Safety Incidents in Korea: a Cross-Sectional Study. J Korean Med Sci 2020; 35:1-11. https://doi.org/10.3346/jkms.2020.35.e118

25. Choi EY, Pyo J, Lee W, et al. Nurses' experiences of patient safety incidents in Korea: a cross-sectional study. BMJ Open 2020; 10:1-11 https://doi.org/10.1136/bmjopen-2020$\underline{037741}$ 